\title{
Platelet membrane lipid rafts protein composition varies following GPVI and
}

\section{CLEC-2 receptors activation}

Irene Izquierdo ${ }^{1}$, María N. Barrachina ${ }^{1}$, Lidia Hermida-Nogueira ${ }^{1}$, Vanessa Casas ${ }^{2}$, Johannes A. Eble ${ }^{3}$, Montserrat Carrascal², Joaquín Abián ${ }^{2}$ and Ángel García ${ }^{1 *}$

${ }^{1}$ Platelet Proteomics Group. Center for Research in Molecular Medicine and Chronic Diseases (CIMUS), Universidad de Santiago de Compostela, and Instituto de Investigación Sanitaria (IDIS), Santiago de Compostela, Spain.

${ }^{2}$ CSIC/UAB Proteomics Laboratory, IIBB-CSIC-IDIBAPS, Barcelona, Spain.

${ }^{3}$ Institute of Physiological Chemistry and Pathobiochemistry, University of Münster, Münster, Germany.

\section{*Corresponding author:}

Dr. Ángel García

Center for Research in Molecular Medicine and Chronic Diseases (CIMUS).

Universidade de Santiago de Compostela.

Avda. Barcelona s/n

15782- Santiago de Compostela (Spain)

Email: angel.garcia@usc.es 


\begin{abstract}
Lipid rafts are membrane microdomains that have been proposed to play an important role in several platelet-signalling cascades, including those mediated by the receptors Glycoprotein VI (GPVI), and C-type lectin domain family 1 member B (CLEC-2), both involved in thrombus formation. We have performed a LC-MS/MS proteomic analysis of lipid rafts isolated from platelets activated through GPVI and CLEC-2 as well as from resting platelets. Our aim was to determine the magnitude of changes in lipid rafts protein composition and to elucidate the relevance of these alterations in platelet function. A number of relevant signalling proteins were found enriched in lipid rafts following platelet activation (such as the tyrosine protein kinases Fyn, Lyn and Yes; the G proteins $\mathrm{G}(\mathrm{i})$ and $\mathrm{G}(\mathrm{z})$; and cAMP protein kinase). Interestingly, our results indicate that the relative enrichment of lipid rafts in these signalling proteins may not be a consequence of protein translocation to these domains upon platelet stimulation, but the result of a massive loss in cytoskeletal proteins after platelet activation. Thus, this study may help to better understand the effects of platelet activation in the reorganization of lipid rafts and set the basis for further proteomic studies of these membrane microdomains in platelets.
\end{abstract}


Keywords: Platelets, lipid rafts, GPVI, CLEC-2, LC-MS/MS

Significance: We performed the first proteomic comparative analysis of lipid raftsprotein composition in platelets activated through GPVI and CLEC-2 receptors and in resting state. We identified a number of signalling proteins essential for platelet activation relatively enriched in platelets activated through both receptors, and we show that lipid rafts reorganization upon platelet activation leads to a loss in cytoskeletal proteins, highly associated to these domains in resting platelets. 


\section{Introduction}

Lipid rafts, also known as glycolipid-enriched membrane (GEMs) domains, are liquidordered membrane microdomains highly enriched in sphingolipids and cholesterol [1]. These membrane structures display lateral mobility in the cell membrane and are known to contain key signalling proteins in numerous cell types $[2,3]$.

In platelets, lipid rafts represent a prolific field of study since they have been proposed to participate in crucial signalling events involving platelet activation [4]. Platelet lipid rafts have been widely investigated over the last decades in order to determine their relevance in platelet signalling through different platelet receptors, including Glycoprotein VI (GPVI) and C-type lectin like receptor 2 (CLEC-2) [5-8]. Remarkably, numerous studies have reported that these receptors play an essential role in thrombus formation triggered by unwanted platelet activation [9-12].

GPVI is a membrane receptor exclusively expressed in megakaryocytes and platelets where it acts as the major receptor for collagen. CLEC-2 is highly expressed on platelet surface and it can be activated by its interaction with the endogenous protein podoplanin [13] and with the snake venom protein rhodocytin [14].

GPVI- mediated platelet activation requires its non-covalent interaction with the Fc receptor $\gamma$ chain $(\mathrm{FcR} \gamma)$ and the phosphorylation of its immunoreceptor tyrosinebased activation motif (ITAM) [15]. Similarly, the CLEC-2 signalling pathway is triggered by the phosphorylation of a hemITAM motif on its cytosolic domain [16]. The downstream activation cascades of both receptors show important resemblances, including the early participation of the tyrosine-protein kinase Syk and subsequent phosphorylation of the adapter LAT culminating in the activation of PLC $\gamma 2[14,17]$. 
The crucial role of lipid rafts in orchestrating the downstream events that occur upon GPVI and CLEC-2 activation has been controversial over the last years. Locke et al. showed a massive recruitment of GPVI to lipid rafts when platelets were activated with the GPVI agonist convulxin [7], whereas Wonerow et al. found no major change on GPVI association to rafts upon platelet stimulation [18]. Regarding CLEC-2, Pollitt et al. proposed a model in which lipid rafts play an essential role in the activation of the signalling pathway mediated by this receptor [6] while, in a recent study, Manne et al. reported that the diminished functional responses after lipid rafts disruption could be attributed to the attenuated effects of $G_{i}$ signalling [19].

In this study, we aimed to analyse the protein content of lipid rafts from resting and activated platelets, and thus determine the magnitude of changes in protein composition that occur when platelets are activated through GPVI and CLEC-2 receptors. This was done by a proteomic approach using liquid chromatography coupled with high-resolution mass spectrometry together with label free quantitation.

\section{Materials and methods}

The workflow of the experimental approach is shown in Figure 1.

\subsection{Platelet isolation and activation}

Fresh blood samples were collected from healthy volunteers in coagulation $3,2 \%$ sodium citrate tubes (Vacuette) and processed within the first 90 minutes after extraction.

Platelets were isolated by an established method designed to limit contamination from other blood cells and plasma proteins [20]. Finally, washed platelets were resuspended in HEPES-Tyrodes $\left(134 \mathrm{mmol} / \mathrm{L} \mathrm{NaCl}, 0.34 \mathrm{mmol} / \mathrm{L} \quad \mathrm{Na}_{2} \mathrm{HPO}_{4}, 2.9\right.$ 
mmol/L KCl, $12 \mathrm{mmol} / \mathrm{L} \mathrm{NaHCO}_{3}, 20 \mathrm{mmol} / \mathrm{L}$ HEPES, $5 \mathrm{mmol} / \mathrm{L}$ glucose, $1 \mathrm{mmol} / \mathrm{L}$ $\mathrm{MgCl}_{2}, \mathrm{pH}$ 7.3) at the desired concentration of $1,25 \times 10^{9}$ platelets $/ \mathrm{ml}$ and allowed to rest for 30 minutes at room temperature.

Activations were performed with $500 \mu \mathrm{l}$ of washed platelets in a Chrono-log aggregometer. For GPVI- mediated platelet activation, platelets were incubated at $37^{\circ} \mathrm{C}$ with $3 \mu \mathrm{g} / \mathrm{ml}$ of the GPVI- specific agonist Collagen- related peptide (CRP-XL) for 90 seconds. CRP, defined as a $\mathrm{GCO}-(\mathrm{GPO})_{10}-\mathrm{GCOG}$ sequence peptide (where $\mathrm{G}$ is Glycine, $\mathrm{C}$ is Cystein, $\mathrm{P}$ is Prolin, and $\mathrm{O}$ is Hydroxiprolin), acts as a potent GPVI agonist when it is cross-linked (CRP-XL) forming a quaternary structure [21]. For CLEC-2- mediated platelet activation, platelets were incubated at $37^{\circ} \mathrm{C}$ with the CLEC2 agonist rhodocytin at a concentration of $200 \mathrm{nM}$ for 5 minutes. Resting platelets were allowed to incubate at $37^{\circ} \mathrm{C}$ in absence of platelet agonists for the maximum activation time (5 minutes). All activations were performed after the addition of $9 \mu \mathrm{M}$ Integrilin ${ }^{\circledR}$ (GlaxoSmithKline) in order to avoid platelet aggregation and the activation of $\alpha \operatorname{IIb} \beta 3$ signalling cascade. Optimal agonists' concentrations and activation times were previously determined by dose-response and time course biochemical assays measuring protein tyrosine phosphorylation levels upon receptor activation (data not shown).

\subsection{Membrane fractions isolation by sucrose gradient ultracentrifugation}

After activation or incubation, platelets' membranes were disrupted in ice-cold lysis buffer (25mM Tris-HCL [pH 7.6], 150mM NaCl, 5mM EDTA, 1\% Brij 58, protease inhibitors cocktail mammalian M250-1ML VWR). The same buffer was used to obtain the sucrose gradient solutions.

Platelet lysates $(1 \mathrm{ml})$ were mixed with an equal volume of $80 \% \mathrm{w} / \mathrm{v}$ sucrose, leading to a final concentration of $40 \% \mathrm{w} / \mathrm{v}$ sucrose, and loaded on ultracentrifuge tubes 
(SW 55 Ti Ultra-Clear, Beckman Coulter). The lysates were overlaid with $2 \mathrm{ml}$ of $30 \%$ w/v sucrose followed by $1 \mathrm{ml}$ of $5 \%$ w/v sucrose.

Membrane fractions were separated by ultracentrifugation at $48000 \mathrm{~g}$ and $4^{\circ} \mathrm{C}$ for 16h (SW 55 Ti rotor, Beckman Coulter Optima L-100XP ultracentrifuge). After ultracentrifugation, 10 fractions of $500 \mu \mathrm{l}$ were collected from each sample and supplemented with $1 \% \mathrm{~N}$-dodecyl- $\beta$-D-gluco-maltoside to solubilize the lipid rafts.

\subsection{Western Blotting}

A lysate was obtained from each fraction by dissolving $32 \mu \mathrm{l}$ of sample in Laemmli sample buffer (final composition $2 \% \mathrm{w} / \mathrm{v}$ SDS, $5 \%$ v/v 2-mercaptoethanol, $10 \%$ v/v glycerol, $25 \mathrm{mM}$ Tris, $\mathrm{pH} 6.8$ ). After boiling the lysates for 5 minutes at $99^{\circ} \mathrm{C}$, they were loaded on NuPAGE 4-12\% Bis-Tris protein gels. Following electrophoresis, performed at $120 \mathrm{~V}$ in an XCell SureLock ${ }^{\mathrm{TM}}$ Mini-Cell system (Invitrogen), proteins were transferred onto polyvinylidene difluoride (PVDF) membranes (GE Healthcare) by using an XCell $\mathrm{II}^{\mathrm{TM}}$ Blot Module, and allowing proteins to transfer to the membrane for 2 hours at $200 \mathrm{~mA}$ and $4^{\circ} \mathrm{C}$.

Membranes were blocked in 5\% BSA in TBS-T (20 mM Tris- $\mathrm{HCl}$ (pH 7.6), 150 $\mathrm{mM} \mathrm{NaCl}$ and $0.1 \%$ Tween 20 ) overnight at $4^{\circ} \mathrm{C}$. Once blocked, they were incubated for 90 minutes at room temperature with the corresponding antibody (mouse antiFlotillin-1 antibody (sc-74566, Santa Cruz Biotechnology) dilution 1/500; rabbit antiIntegrin $\alpha \mathrm{IIb}$ antibody (sc-15328, Santa Cruz Biotechnology) dilution 1/500; rabbit antiLyn antibody (R.677.10, Invitrogen, Thermo Fisher Scientific) dilution 1/1000; or mouse anti- Actin antibody (MAB1501, Merck Millipore) dilution 1/1000).

Following washes in TBS-T, membranes were exposed to horseradish peroxidase labelled goat anti- mouse antibody 31430 or goat anti- rabbit antibody 31460 
(Pierce, Rockford, IL) dilution 1/5000. Membranes were washed again and processed using an enhanced chemiluminiscence system (ECL, Pierce, Rockford, USA).

Bands intensity was quantified by densitometry using ImageJ (National Institute of Health, Bethesda, MD, USA) version 1.47.

\subsection{Lipid rafts protein isolation by ultracentrifugation}

Fractions that showed enrichment in the lipid rafts marker Flotillin-1 and that lacked integrin $\alpha$ IIb were considered lipid rafts- enriched fractions. This assumption is based on the previous knowledge that membrane lipid rafts contain a wide amount of Flotillin1 [22] while lacking integrin $\alpha \mathrm{IIb}[6]$.

These fractions were diluted 1/3 with gradient buffer (25mM Tris-HCL [pH 7.6], 150mM NaCl, 5mM EDTA, protease inhibitors cocktail mammalian M250-1ML VWR) and ultracentrifuged at $100000 \mathrm{~g}$ and $4^{\circ} \mathrm{C}$ for $1 \mathrm{~h}$ (SW 55 Ti rotor, Beckman Coulter Optima L-100XP ultracentrifuge). Pellets were lysed in 2\% SDS, $500 \mathrm{mM}$ Tris pH 7.6, 0.05 M DTT.

\subsection{Protein digestion and LC-MS/MS protein identification}

The most suitable protein digestion method for processing these samples was selected in a first approach. Briefly, four samples corresponding to lipid rafts enriched fractions isolated from $6.25 \cdot 10^{8}$ platelets were processed separately. Two samples (technical replicates) were digested with trypsin following filter aided sample preparation (FASP) method [23] and the other two samples were in-gel trypsin- digested. Prior to performing in-gel digestion, samples were supplemented with $5 \%$ v/v 2 mercaptoethanol and $10 \% \mathrm{v} / \mathrm{v}$ glycerol (final concentration), and were allowed to move 
into the resolving phase of a $12 \%$ SDS-PAGE gel. Then, gels were fixed with $10 \% \mathrm{v} / \mathrm{v}$ methanol and 7\% v/v acetic acid prior to Sypro Ruby staining. Sypro Ruby- stained bands were excised and digested using an automatic device (DigestPro MS, Intavis, Cologne, Germany). The process involved reduction with DTT, derivatization with iodoacetamide, and enzymatic digestion with trypsin $\left(37^{\circ} \mathrm{C}, 8 \mathrm{~h}\right)$.

The tryptic digests obtained from both procedures were dried and dissolved in $44 \mu \mathrm{l}$ of methanol/water/trifluoroacetic acid (5/95/0.1). Fifty percent of the sample (22 $\mu \mathrm{l})$ was loaded in the chromatographic system, consisting in a C18 preconcentration cartridge (Agilent Technologies) connected to a $15 \mathrm{~cm}$ long, $100 \mu \mathrm{m}$ i.d. C18 column (Nikkyo Technos Co.). The separation was done at $0.4 \mu \mathrm{L} / \mathrm{min}$ in a $120-\mathrm{min}$ acetonitrile gradient from 3 to $35 \%$ (solvent A: $0.1 \%$ formic acid, solvent B: acetonitrile $0.1 \%$ formic acid). The HPLC system was composed of an Agilent 1200 capillary nano pump, a binary pump, a thermostated micro injector and a micro switch valve. The LTQ XL Orbitrap was operated in the positive ion mode with a spray voltage of $1.8 \mathrm{kV}$. The spectrometric analysis was performed in a data dependent mode, acquiring a full scan followed by $10 \mathrm{MS} / \mathrm{MS}$ scans of the 10 most intense signals detected in the MS scan from the global list. The full MS (range 400-1650) was acquired in the Orbitrap with a resolution of 60.000. The MS/MS spectra were done in the linear ion-trap. The spectra were searched using SEQUEST (Proteome Discoverer v1.4, ThermoFisher) with the following parameters: peptide mass tolerance $20 \mathrm{ppm}$, fragment tolerance 0.6 Da, enzyme set as Trypsin and allowance of up to 3 missed cleavages, dynamic modification of methionine oxidation $(+16 \mathrm{Da})$, and fixed modification of cysteine carbamidomethylation (+57 Da). The database used for searching was UniProt Homo Sapiens 16_01. Final results were filtered by peptide rank 1 and peptide confidence high (1\% FDR). 
For lipid rafts comparative analysis, four biological replicates were processed for each condition (Basal, GPVI, and CLEC-2). Lipid rafts protein samples were obtained from $6.25 \cdot 10^{8}$ platelets by following the aforementioned ultracentrifugation steps and the whole protein samples recovered after ultracentrifugation were digested by FASP. Tryptic digests were redissolved in $5 \mu \mathrm{l}$ of methanol/water/trifluoroacetic acid and $40 \%$ of the sample was diluted to $20 \mu$ prior to its loading in the chromatographic system. The separation was done at $0.4 \mu \mathrm{L} / \mathrm{min}$ in a 180 -min acetonitrile gradient from 3 to $35 \%$ and the subsequent spectrometric analysis and spectra search was performed as mentioned above.

\subsection{Ingenuity pathways and database analysis}

Ingenuity Pathways Analysis (IPA) software (Ingenuity Systems, CA, USA) was used to determine the protein enrichment in relevant pathways and biological functions. Only proteins that were detected in at least two of the four biological replicates analysed per condition were considered for the analysis.

The mammalian lipid raft proteome database RaftProt [24] was consulted in order to inquire if the identified proteins were previously found associated to lipid rafts.

UniProt database [25] was used to obtain information about protein function and subcellular location.

\section{Results}

\subsection{Resting and activated platelets show the same pattern of lipid rafts enrichment among density gradient fractions obtained by ultracentrifugation}

The determination of lipid rafts- enriched fractions was performed by Western Blot. We considered as lipid rafts enriched fractions those that contained the lipid rafts- marker 
Flotillin-1 while lacked Integrin $\alpha \mathrm{IIb}$, which is known to be expressed all along the cell membrane. Fraction 2 was selected for being analysed by LC-MS/MS in all conditions and biological replicates, since it was the one that best met the established criteria (Figure 2).

\subsection{FASP digestion is the most suitable procedure for LC-MS/MS analysis of lipid rafts- protein samples}

Prior to the quantitative study, we performed a preliminary proteomic analysis of proteins isolated from lipid rafts from resting platelets following two different protein digestion methods: FASP and in-gel trypsin digestion.

Two technical replicates were performed per digestion method. In the case of ingel digestion, the sample was concentrated in a single gel band, which was then excised and submitted to digestion and LC-MS/MS analysis.

The total number of proteins identified was of 447 (295 identifications in in-gel digested samples and 281 in FASP-digested samples, being 129 common to both approaches). Although there is a slight increase in the number of identified proteins when samples are in-gel digested, the distribution of protein functions is very similar among the two sets of proteins (Figure 3A). Nevertheless, in-gel digested samples were found to recover more mitochondrion proteins than in-solution digested samples. The latter were even more abundantly found than membrane proteins in the whole geldigested samples (Figure 3B). Mitochondrion proteins are usually recovered together with cell membrane lipid rafts proteins. Nonetheless, when trying to analyse cell membrane lipid rafts, a lower number of isolated mitochondrion proteins is preferred in order to better analyse the composition of plasma membrane lipid rafts. 
Further, we looked upon the identified proteins that are known to be associated to lipid rafts in platelets. Thereby, we found that these proteins (i.e. the lipid raftsmarkers Flotillins 1 and 2) were clearly most efficiently recovered when using the FASP digestion procedure (Table 1).

Considering the above, we conclude that FASP digestion is the more reliable method for analysing the protein composition of platelet lipid rafts samples.

\subsection{Overall results from the LC-MS/MS analysis}

A total number of 247 proteins were identified among all conditions and biological replicates excluding contaminants (mostly keratins) from the results. (Figure 4A) (see

\section{Supplementary table 1).}

In order to compare the relative abundance of the identified proteins among the three conditions, we calculated the normalized number of PSMs (Peptide Spectrum Matches) for each sample. To do the latter, we obtained the quotient between the number of PSMs for a single protein and the total number of PSMs of the sample. This calculation provides information that more precisely represents the relative abundance of each protein in the sample. Proteins included in the analysis were those identified by at least two PSMs. Information on the absolute and the relative abundance of these proteins among conditions can be found in Supplementary table 2. Fold changes were calculated as the quotient of the normalized number of PSMs between conditions.

To achieve higher consistency in the results of the comparative analysis between conditions, we focused on proteins that were identified in at least two of the four biological replicates for each condition. After applying this filter to the overall results, we identified 51 proteins in basal samples, 52 in GPVI samples and 36 in CLEC-2 
samples. From these, 31 proteins were common to the three conditions (Figure 4B) indicating a slight difference in the variety of proteins that associate to lipid-rafts before and after platelet activation. Interestingly, $92 \%$ of the proteins were previously identified associated to lipid rafts according to RaftProt database.

From the proteins included in our comparative analysis (those that were identified in at least two of the four biological replicates), the vast majority are known to participate in cell signalling and cytoskeleton-remodelling processes (Figure 5A). Regarding protein location, most proteins are expressed on the cell membrane and cell surface, as expected. Remarkably, a higher amount of proteins associated to the mitochondrion was found in activated platelets through both GPVI and CLEC-2 receptors, whereby this increase was more noticeable in GPVI- activated samples (Figure 5B).

\subsection{Lipid rafts from platelets activated through both GPVI and CLEC-2 receptors are enriched in key platelet signalling proteins}

Interestingly, some proteins that are known to be essential for platelet activation, and that were previously described to be associated with lipid rafts, were found relatively enriched in these membrane domains when platelets were activated through CLEC-2 and GPVI receptors. Figure 6 shows the relative abundances of relevant signalling proteins enriched in lipid rafts from platelets activated with the CLEC-2 agonist rhodocytin (Figure 6A), or with the GPVI agonist CRP-XL (Figure 6B) (Fold change of normalized number of PSMs > 2). Some of these proteins were found relatively enriched in lipid rafts fractions from platelets activated through both receptors. These proteins include: the tyrosine kinase Lyn (LYN_HUMAN); the tyrosine kinase Yes (YES_HUMAN); the Guanine nucleotide-binding protein G(i) (GNAI2_HUMAN); the 
Guanine nucleotide-binding protein G(z) (GNAZ_HUMAN); the alpha and beta regulatory subunits of cAMP-dependent protein kinase (KAPCA_HUMAN; KAPCB_HUMAN); the catalytic subunit of cAMP-dependent protein kinase (KAPC0_HUMAN); the voltage- dependent anion channel 3 (VDAC3_HUMAN); and the voltage- dependent anion channel 1 (VDAC1_HUMAN). Furthermore, CD59 glycoprotein (CD59_HUMAN) was solely identified in activated samples, and the proper receptor GPVI was found to be relatively enriched in lipid rafts from CRPactivated platelets.

\subsection{Lyn enrichment in lipid rafts from activated platelets may be a consequence of the loss of cytoskeletal proteins in these domains}

Since our LC-MS/MS results suggested that, upon platelet stimulation through CLEC-2 and GPVI receptors, an enrichment in key signalling proteins in lipid rafts occurs, we decided to validate our findings in samples obtained from a different cohort of four healthy donors.

Western blots performed against the tyrosine kinase Lyn showed no differences in the percentage of the protein associated to the main raft fraction among the three analysed conditions ( $43 \pm 8.1 \%$ for basal samples; $40 \pm 8.6 \%$ for GPVI- activated; and 39 $\pm 12.4 \%$ for CLEC-2-activated; Figure 7). To clarify this apparent contradiction between proteomic and western blot data in the case of Lyn, we decided to investigate if there was a potential loss of any other proteins from lipid rafts in activated platelets, which could cause a relative enrichment of Lyn and other signalling proteins in these fractions upon platelet stimulation. Since we found different isoforms of Actin (ACTBL_HUMAN, ACTG_HUMAN, and ACTS_HUMAN), and further cytoskeletal proteins (such as Myosin-9; MYH9_HUMAN), highly associated to lipid rafts in basal 
samples in our LC-MS/MS analysis (association that was diminished in activated platelets), we decided to determine the pattern of enrichment of Actin among differential density fractions in the three analysed conditions. Indeed, we found by western blot an important percentage of actin $(20 \pm 9.2 \%)$ co-localizing with the lipid rafts marker Flotillin-1 in samples obtained from resting platelets, but this association is lost when platelets are activated through both GPVI and CLEC-2 receptors (Figure 7). Our results suggest that, in resting platelets, an important fraction of the actin cytoskeleton is associated to membrane rafts, basing on the number of proteins identified and their relative abundances (see Supplementary table 2) but, upon GPVI or CLEC-2 stimulation, lipid rafts reorganization and cytoskeleton remodelling triggers the loss of the majority of the cytoskeletal components in these membrane domains.

\subsection{Enriched canonical pathways and biological functions}

A core analysis powered by IPA software was performed for each condition including the set of proteins identified in at least two of the four biological replicates analysed.

A comparative analysis between conditions showed a similar pattern of enrichment in most relevant canonical pathways and biological functions (Figure 8).

\section{Discussion}

Platelet lipid rafts have been deeply studied over the last decades due to their wide content in signalling proteins essential for platelet function [26-28]. More precisely, a number of researchers have proposed that signalling following GPVI and CLEC-2 receptors- activation is highly dependent on lipid rafts $[6,7,17]$. Locke et al. found no GPVI associated to lipid rafts in resting platelets but a massive recruitment to these 
membrane fractions following its stimulation [7]. Regarding CLEC-2, Pollitt et al. proposed a model for platelet activation through this receptor in which lipid rafts play an crucial role [6]. Nevertheless, there has been controversy on the requirement of lipid rafts for platelet activation orchestrated by these receptors. Thus, Wonerow et al. found a considerable amount of GPVI associated to rafts in resting platelets with no major variation upon GPVI- stimulation [18]. This discrepancy can be attributed to the dissimilarities in lipid rafts- isolation procedures. Locke et al. employed Triton X-100 as non-ionic detergent for platelet lysis, while Wonerow et al. used Brij ${ }^{\circledR} 58$, suggesting a weak association of GPVI with lipid rafts in resting platelets that is more preserved when Brij ${ }^{\circledR} 58$ is utilized to disrupt platelet membrane. Regarding CLEC-2, Manne et al. found that a great amount of CLEC-2 remains on the membrane soluble fractions upon its stimulation although a small proportion moves to lipid rafts. Furthermore, they reported that the primary signalling events downstream of CLEC-2 do not require raft environment and that the diminished responses seen when these domains are disrupted can be attributed to the attenuated effects of $G_{i}$ signalling.

In this study, we performed a LC-MS/MS proteomic analysis of lipid rafts isolated from platelets activated through GPVI and CLEC-2 and resting platelets. Our aim was to determine the extent on lipid rafts- protein content changes upon these receptors stimulation.

We followed a well stablished protocol for lipid rafts isolation based on the employment of the non-ionic detergent Brij ${ }^{\circledR} 58$ [6]. This detergent, mentioned above, was found to preserve these microdomains even when used at high concentrations and it was also found to be more efficient than Triton X-100 in maintaining the association of GPVI to lipid rafts in resting platelets [7, 18]. 
Most proteins identified in our LC-MS/MS analysis are related to cell signalling and cytoskeleton-remodelling events necessary for platelet function. This observation strengthens the idea of the requirement of lipid rafts for signal transduction as well as their role in the cross talk between membrane proteins and the cytoskeleton $[8,29,30]$.

Lipid rafts enriched fractions from platelets activated through CLEC-2 and, specially, GPVI present a higher content in mitochondrial proteins than those isolated from resting platelets (such as MICOS complex subunit MIC19). The identification of typical mitochondrial proteins when analysing lipid rafts samples can be attributed either to their expression on these cell membrane domains or to the presence of lipid rafts-like domains in mitochondrion, which leads to the co-isolation of these proteins together with lipid rafts [31]. Although it has to be taken cautiously, as their presence in lipid rafts samples might be a contamination, the identification of mitochondrial proteins in lipid rafts fractions could be explained by the recruitment of molecules with an essential role in energetic metabolism to these domains when platelet- energetic requirements rise due to platelet activation [32-34]. Conversely, lipid rafts from resting platelets were found to be associated to a larger amount of some cytoskeletal proteins, such as Myosin-9 and different isoforms of Actin.

A number of key platelet signalling proteins (tyrosine kinases Lyn, Fyn and Yes; $\mathrm{G}$ protein $\mathrm{G}_{\mathrm{i}}$ and the cAMP-dependent protein kinase) were identified in all analysed samples showing a pattern of relative enrichment favourable to both activation conditions. These observations support the previously reported reorganization of lipid rafts upon platelet stimulation leading to their enrichment in proteins essential for adequate activation of these platelet-signalling cascades. Nevertheless, when we examined the percentage of the tyrosine kinase Lyn associated to lipid rafts, we found no differences between conditions, indicating that its relative enrichment in these 
domains may be the consequence of the loss of some other proteins when platelets are activated. Thus, we analysed the distribution of Actin among the density gradient platelet fractions, and observed that an important percentage of this protein is associated to lipid rafts in resting platelets, but this association is lost when platelets are activated with GPVI or CLEC-2 agonists. These results suggest that membrane rafts reorganization together with platelet cytoskeleton remodelling processes, which occur during platelet stimulation, lead to a relative enrichment in key signalling proteins in these domains and to the loss of a wide amount of cytoskeletal components. Further research is needed to investigate in detail the biochemical reasons for this observation and its functional implications.

Interestingly, both Voltage-dependent anion-selective channel proteins 1 and 3 (VDAC1 and VDAC3) were found in a higher relative amount in activated platelets. These proteins are mitochondrial porins but were reported to be expressed in the plasma membrane associated with lipid rafts, where they have been related to cellular ATP release, calcium and metabolite transport, and volume control, among other functions [35]. The relative enrichment of these proteins in lipid rafts from activated platelets might indicate that these porins could be playing a role in the membrane trafficking events that occur when platelets are activated though CLEC-2 and GPVI receptors.

As expected, a higher amount of GPVI was isolated in lipid rafts from GPVIactivated platelets, although this collagen receptor was also recovered from basal samples. This observation confirms that GPVI is partially associated to lipid rafts in resting platelets but GPVI activation leads to its enrichment in these membrane fractions.

CLEC-2 receptor was not identified in lipid raft- enriched fractions from any platelet stimulation conditions. This is consistent with the previously reported remark 
that, although a small proportion of CLEC-2 translocates to lipid rafts upon rhodocytinstimulation, the great amount of this receptor remains in the soluble fractions of the platelet membrane [19].

The protein CD59 was found exclusively in lipid rafts isolated from activated platelets through both GPVI and CLEC-2, although with a low number of PSMs. CD59 is a glycoprotein expressed on the cell membrane that has been reported to play a role in T-cell activation after the stimulation of T-cell receptor (TCR) [36]. TCR signals trough the phosphorylation of its ITAMs motifs by the Src family kinase Lck [37-39]. This similarity with the primary signalling events downstream of the activation of GPVI and CLEC-2, and its possible recruitment to lipid rafts upon platelet activation, suggest a potential role of this protein in the signalling cascades underlying these receptors.

\section{Study limitations}

This is a semi-quantitative proteomic study in which we reported a considerable amount of typical mitochondrial proteins in our samples. Although their identification could be attributed to their expression in the plasma membrane, their presence in lipid rafts samples could be the result of their co-isolation due to the similar physical properties of some mitochondrial proteins and lipid rafts proteins. It is also important to highlight that precipitation steps were avoided during protein isolation to maximize protein recovery and diminish technical variability. Nevertheless, this approach may not lead to a complete protein recovery; however, all samples were processed in parallel in the same way to guarantee comparisons were accurate.

\section{Conclusion}

In conclusion, we performed the first proteomic analysis of platelet- lipid rafts comparing resting state with two stimulation conditions. Our results reveal the 
complexity of lipid rafts protein composition and their relative enrichment in a number of relevant signalling proteins, together with a loss in cytoskeletal proteins, following platelet activation through GPVI and CLEC-2 receptors. These results may help to better understand the role of lipid rafts in platelet activation and set the basis for further biochemical and functional studies of these membrane microdomains in platelets.

\section{Funding}

This work was supported by the Spanish Ministry of Economy and Competitiveness (MINECO) [grant No. SAF2016-79662-R], co-funded by the European Regional Development Fund (ERDF); the Consellería de Cultura, Educación e Ordenación Universitaria, Xunta de Galicia [predoctoral grant Plan I2C 2014; and Centro Singular de investigación de Galicia accreditation 2016-2019, ED431G/05; both co-funded by the ERDF]. J.A.E. is supported by Deutsche Forschungsgemeinschat [DFG grant: EB177/13-1].

\section{Acknowledgements}

The authors would like to thank the personnel from the Servizo de Vixilancia da Saúde, Universidade de Santiago de Compostela, for their assistance on blood collection from healthy volunteers. We would like to thank Prof. Richard Farndale, from the University of Cambridge, for suppling CRP-XL. The Proteomics Laboratory CSIC/UAB is a member of Proteored, PRB3-ISCIII, and is supported by Grant PT17/0019/0008, funded by ISCIII and FEDER.

\section{Conflict of interest}

The authors declare no conflict of interest 


\section{References}

[1] Simons K, Ikonen E. Functional rafts in cell membranes. Nature 1997; 387(6633):569-72

[2] Rietveld A, Simons K. The differential miscibility of lipids as the basis for the formation of functional membrane rafts. Biochim Biophys Acta 1998; 1376(3):467-79

[3] Brown DA, London E. Functions of lipid rafts in biological membranes. Annu Rev Cell Dev Biol 1998; 14:111-36

[4] Bodin S, Tronchère H, Payrastre B. Lipid rafts are critical membrane domains in blood platelet activation processes. Biochim Biophys Acta 2003, 1610(2):247-57

[5] Quinton TM, Kim S, Jin J, Kunapuli SP. Lipid rafts are required in Galpha(i) signaling downstream of the P2Y12 receptor during ADP-mediated platelet activation. $\mathrm{J}$ Thromb Haemost 2005; 3(5):1036-41

[6] Pollitt AY, Grygielska B, Leblond B, Désiré L, Eble JA, Watson SP. Phosphorylation of CLEC-2 is dependent on lipid rafts actin polymerization secondary mediators and Rac. Blood 2010; 115(14):2938-46

[7] Locke D, Chen H, Liu Y, Liu C, Kahn M. Lipid rafts orchestrate signaling by the platelet receptor glycoprotein VI. J Biol Chem 2002; 277(21):18801-9

[8] Bodin S, Soulet C, Tronchère H, Sié P, Gachet C, Plantavid M, Payrastre B. Integrin-dependent interaction of lipid rafts with the actin cytoskeleton in activated human platelets. J Cell Sci 2005; 118(Pt 4):759-69

[9] Watson SP, Herbert JM, Pollitt AY. GPVI and CLEC-2 in hemostasis and vascular integrity. J Thromb Haemost 2010; 8(7):1456-67

[10] Arthur JF, Dunkley S, Andrews RK. Platelet glycoprotein VI-related clinical defects. Br J Haematol 2007; 139(3):363-72

[11] Sachs UJ, Nieswandt B. In vivo thrombus formation in murine models. Circ Res 2007; 100(7):979-91

[12] Kuijpers MJ, Schulte V, Bergmeier W, Lindhout T, Brakebusch C, Offermanns S, Fässler R, Heemskerk JW, Nieswandt B. Complementary roles of glycoprotein VI 
and alpha2beta1 integrin in collagen-induced thrombus formation in flowing whole blood ex vivo. FASEB J 2003; 17(6):685-7

[13] Suzuki-Inoue K, Kato Y, Inoue O, Kaneto MK, Mishima K, Yatomi Y, Yamazaki H, Narimatsu H, Ozaki Y. Involvement of the snake toxin receptor CLEC-2, in podoplanin-mediated platelet activation, by cancer cells. J Biol Chem 2007; 282:2599326001

[14] Suzuki-Inoue K, Fuller GL, García A, Eble JA, Pöhlmann S, Inoue O, Gartner TK, Hughan SC, Pearce AC, Laing GD, Theakston RD, Schweighoffer E, Zitzmann N, Morita T, Tybulewicz VL, Ozaki Y, Watson SP. A novel Syk-dependent mechanism of platelet activation by the C-type lectin receptor CLEC-2. Blood 2006; 107(2):542-9

[15] Moroi M, Jung SM. Platelet glycoprotein VI: its structure and function. Thromb Res 2004; 114(4):221-33

[16] Fuller GL, Williams JA, Tomlinson MG, Eble JA, Hanna SL, Pöhlmann S, SuzukiInoue K, Ozaki Y, Watson SP, Pearce AC. The C-type lectin receptors CLEC-2 and Dectin-1 but not DC-SIGN signal via a novel YXXL-dependent signaling cascade. J Biol Chem 2007; 282(17):12397-409

[17] Ozaki Y, Suzuki-Inoue K, Inoue O. Platelet receptors activated via mulitmerization: glycoprotein VI GPIb-IX-V and CLEC-2. J Thromb Haemost 2013; 11 Suppl 1:330-9

[18] Wonerow P, Obergfell A, Wilde JI, Bobe R, Asazuma N, Brdicka T, Leo A, Schraven B, Horejsí V, Shattil SJ, Watson SP. Differential role of glycolipid-enriched membrane domains in glycoprotein VI- and integrin-mediated phospholipase Cgamma2 regulation in platelets. Biochem J 2002; 364(Pt 3):755-65

[19] Manne BK, Badolia R, Dangelmaier CA, Kunapuli SP. C-type lectin like receptor 2 (CLEC-2) signals independently of lipid raft microdomains in platelets. Biochem Pharmacol 2015; 93(2):163-70

[20] García A. Two-dimensional gel electrophoresis in platelet proteomics research. Methods Mol Med 2007; 139:339-53

[21] Kehrel B, Wierwille S, Clemetson KJ, Anders O, Steiner M, Knight CG, Farndale RW, Okuma M, Barnes Mj. Glycoprotein VI Is a Major Collagen Receptor for Platelet 
Activation: It Recognizes the Platelet-Activating Quaternary Structure of Collagen, Whereas CD36, Glycoprotein IIb/IIIa, and von Willebrand Factor Do Not. Blood 1998; 91:491-9

[22] Salzer U, Prohaska R. Stomatin, flotillin-1, and flotillin-2 are major integral proteins of erythrocyte lipid rafts. Blood 2001; 97:1141-3

[23] Wiśniewski JR, Zougman A, Nagaraj N, Mann M. Universal sample preparation method for proteome analysis. Nat Methods 2009; 6(5):359-62

[24] Shah A, Chen D, Boda AR, Foster LJ, Davis MJ, Hill MM. RaftProt: mammalian lipid raft proteome database Nucleic Acids Res 2015; 43(Database issue):D335-8

[25] UniProt Consortium, T. UniProt: the universal protein knowledgebase. Nucleic Acids Res 2018; 46(5):2699

[26] Dorahy DJ, Lincz LF, Meldrum CJ, Burns GF. Biochemical isolation of a membrane microdomain from resting platelets highly enriched in the plasma membrane glycoprotein CD36. Biochem J 1996; 319(Pt 1):67-72

[27] Dorahy DJ, Burns GF. Active Lyn protein tyrosine kinase is selectively enriched within membrane microdomains of resting platelets. Biochem J 1998; 333(Pt 2):373-9

[28] Wonerow P, Watson SP. The transmembrane adapter LAT plays a central role in immune receptor signalling. Oncogene 2001; 20(44):6273-83

[29] Cerecedo D, Martínez-Vieyra I, Maldonado-García D, Hernández-González E, Winder SJ. Association of membrane/lipid rafts with the platelet cytoskeleton and the caveolin PY14: participation in the adhesion process. J Cell Biochem 2015; 116(11):2528-40

[30] Viola A, Gupta N. Tether and trap: regulation of membrane-raft dynamics by actinbinding proteins. Nat Rev Immunol 2007; 7(11):889-96

[31] Garofalo T, Manganelli V, Grasso M, Mattei V, Ferri A, Misasi R, Sorice M. Role of mitochondrial raft-like microdomains in the regulation of cell apoptosis. Apoptosis $2015 ; 20(5): 621-34$ 
[32] Barile CJ, Herrmann PC, Tyvoll DA, Collman JP, Decreau RA, Bull BS. Inhibiting platelet-stimulated blood coagulation by inhibition of mitochondrial respiration. Proc Natl Acad Sci USA 2012; 109(7):2539-43

[33] Ravi S, Chacko B, Sawada H, Kramer PA, Johnson MS, Benavides GA, O'Donnell V, Marques MB, Darley-Usmar VM. Metabolic plasticity in resting and thrombin activated platelets. PLoS One 2015; 10(4):e0123597

[34] Yamagishi SI, Edelstein D, Du XL, Brownlee M. Hyperglycemia potentiates collagen-induced platelet activation through mitochondrial superoxide overproduction. Diabetes 2001; 50(6):1491-4

[35] De Pinto V, Messina A, Lane DJ, Lawen A. Voltage-dependent anion-selective channel (VDAC) in the plasma membrane. FEBS Lett 2010; 584(9):1793-9

[36] Deckert M, Ticchioni M, Mari B, Mary D, Bernard A. The glycosylphosphatidylinositol-anchored CD59 protein stimulates both $\mathrm{T}$ cell receptor zeta/ZAP-70-dependent and -independent signaling pathways in T cells. Eur J Immunol $1995 ; 25(7): 1815-22$

[37] Lipp AM, Juhasz K, Paar C, Ogris C, Eckerstorfer P, Thuenauer R, Hesse J, Nimmervoll B, Stockinger H, Schütz GJ, Bodenhofer U, Balogi Z, Sonnleitner A. Lck mediates signal transmission from CD59 to the TCR/CD3 pathway in Jurkat T cells. PLoS One 2014; 9(1):e85934

[38] Love PE, Hayes SM. ITAM-mediated signaling by the T-cell antigen receptor. Cold Spring Harb Perspect Biol 2010; 2(6):a002485

[39] Palacios EH, Weiss A. Function of the Src-family kinases Lck and Fyn in T-cell development and activation. Oncogene 2004; 23(48):7990-8000 


\section{Figure legends}

Figure 1. Experimental workflow. Schematic representation of the methodology applied and the equipment employed in this study.

Figure 2. Determination of lipid rafts- enriched fractions. (A) Representative antiFlotillin-1 and anti- Integrin $\alpha$ IIb western blots of lysate fractions obtained after sucrose-gradient ultracentrifugation. (B) Densitometry graph representing the distribution of the markers Flotillin-1 and Integrin $\alpha$ IIb among the three conditions in all biological replicates (Mean of four independent experiments).

Figure 3. Function and subcellular location of in-gel and FASP-digested proteins. (A) Pie chart representing the distribution of protein functions between the two different digestion procedures under comparison. (B) Pie chart representing the distribution of protein subcellular locations between the two different digestion procedures.

Figure 4. Proteins identified by LC-MS/MS. Venn diagram representing the total number of proteins that were identified in at least two of the four biological replicates analysed for each condition.

Figure 5. Function and subcellular location of the identified proteins. Pie charts representing $(\mathbf{A})$ the distribution of protein functions and $(\mathbf{B})$ the distribution of protein subcellular locations among the three conditions, considering only those proteins that were identified in at least two of the for biological replicates.

Figure 6. Distribution of relevant signalling proteins enriched in lipid rafts from activated platelets. Summation of normalized PSMs and fold change of proteins that were found relatively enriched in lipid rafts from (A) platelets activated through CLEC2 receptor, and $(\mathbf{B})$ platelets activated trough GPVI receptor (Fold change $\geq 2$ ). Protein 
tags correspond to UniProtKB entry names obviating species references (HUMAN in all cases).

Figure 7. Lyn enrichment in lipid rafts from activated platelets can be explained by the loss of cytoskeletal components. (A) Western Blot images showing Flotillin-1, Lyn, and Actin distribution among lysate fractions obtained after sucrose-gradient ultracentrifugation of platelet lysates. Representative images of four independent experiments. (B) Densitometry graph representing the abundance of Lyn and Actin in the main lipid rafts fraction (co-localizing with the highest amount of Flotillin-1). Mean of four independent experiments.

Figure 8. Ingenuity pathways analysis (IPA). Representation or relevant biological processes and canonical pathways in which the identified proteins are involved. Black line indicates significance threshold. 


\section{Tables}

Table 1. Relevant lipid rafts- related proteins identified by the two different digestion procedures (FASP and in-gel).

\begin{tabular}{|c|c|c|c|c|}
\hline Protein & FASP 1 & FASP 2 & IN-GEL 1 & IN-GEL 2 \\
\hline $\begin{array}{l}\text { Linker for activation of T-cells family } \\
\text { member } 1\end{array}$ & & * & & \\
\hline Flotillin-1 & $*$ & $*$ & & \\
\hline Flotillin-2 & $*$ & $*$ & & \\
\hline Tyrosine-protein kinase Fyn & $*$ & $*$ & & \\
\hline Tyrosine-protein kinase Lyn & $*$ & & & \\
\hline $\begin{array}{l}\text { Guanine nucleotide-binding protein G(i) } \\
\text { subunit alpha-2 }\end{array}$ & $*$ & $*$ & $*$ & $*$ \\
\hline Platelet endothelial cell adhesion molecule & $*$ & $*$ & & \\
\hline $\begin{array}{l}\text { C-type lectin domain family } 1 \text { member B } \\
\text { (CLEC-2) }\end{array}$ & $*$ & $*$ & & $*$ \\
\hline Platelet glycoprotein VI (GPVI) & $*$ & & & \\
\hline Platelet glycoprotein Ib alpha chain & $*$ & $*$ & $*$ & $*$ \\
\hline Platelet glycoprotein Ib beta chain & $*$ & $*$ & $*$ & $*$ \\
\hline Platelet glycoprotein V & $*$ & $*$ & & $*$ \\
\hline $\begin{array}{l}\text { High affinity immunoglobulin epsilon } \\
\text { receptor subunit gamma }\end{array}$ & $*$ & $*$ & $*$ & $*$ \\
\hline
\end{tabular}




\section{Figure}

Click here to download high resolution image

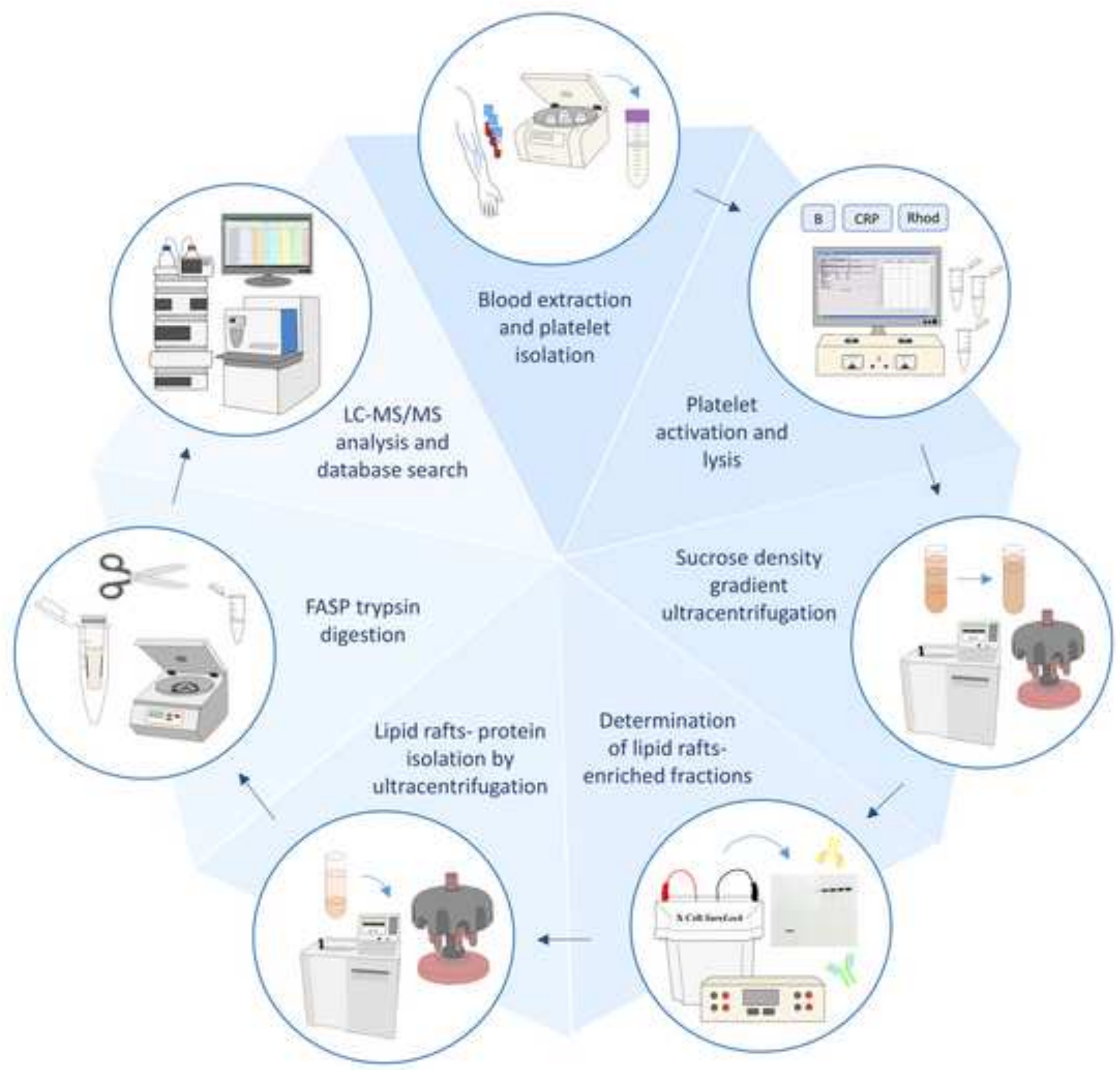


A)

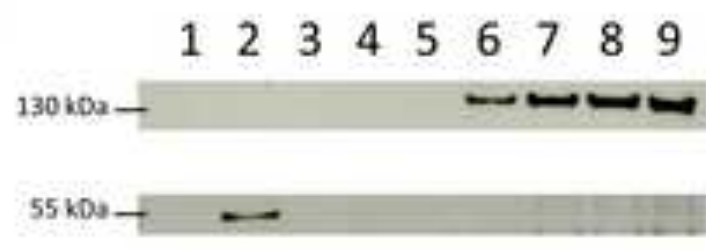

B)

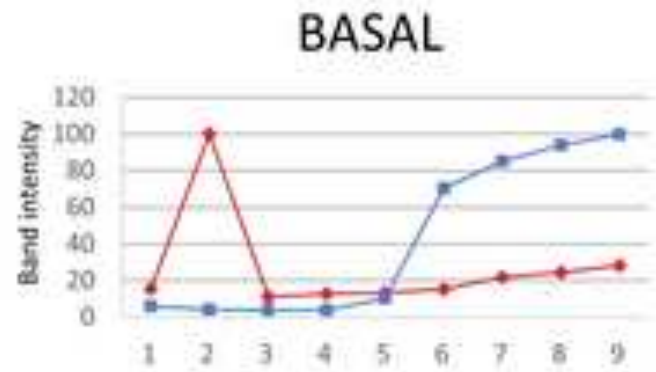

$\begin{array}{lllllllll}12 & 3 & 4 & 5 & 6 & 7 & 8 & 9\end{array}$

$\longrightarrow$

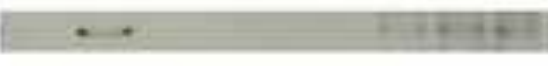

CLEC-2

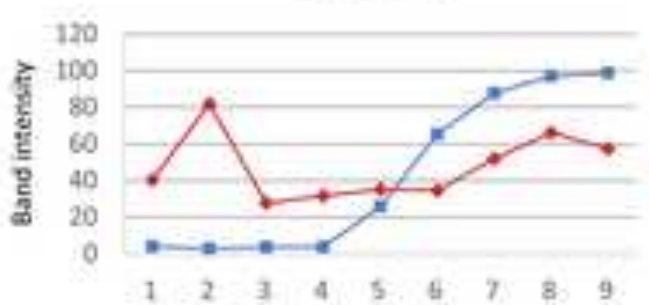

$\begin{array}{lllllllll}1 & 2 & 3 & 4 & 5 & 6 & 7 & 8 & 9\end{array}$ $\longrightarrow$ IB: allB
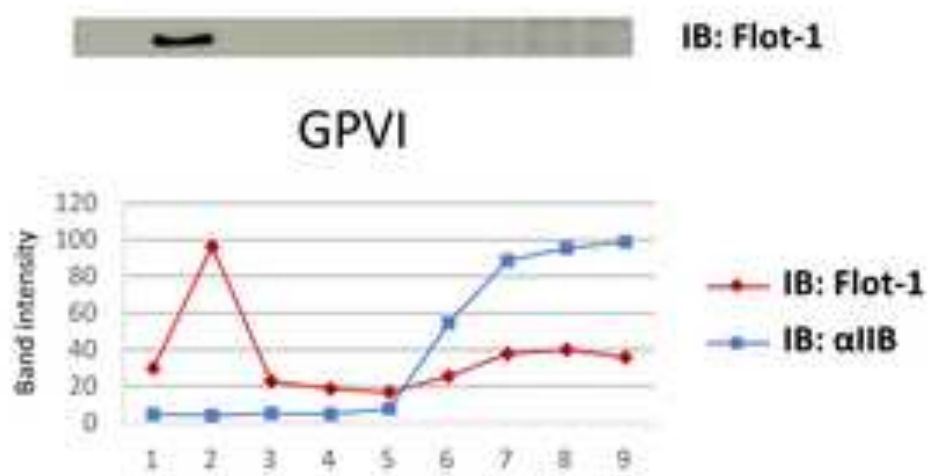
A)

FUNCTION
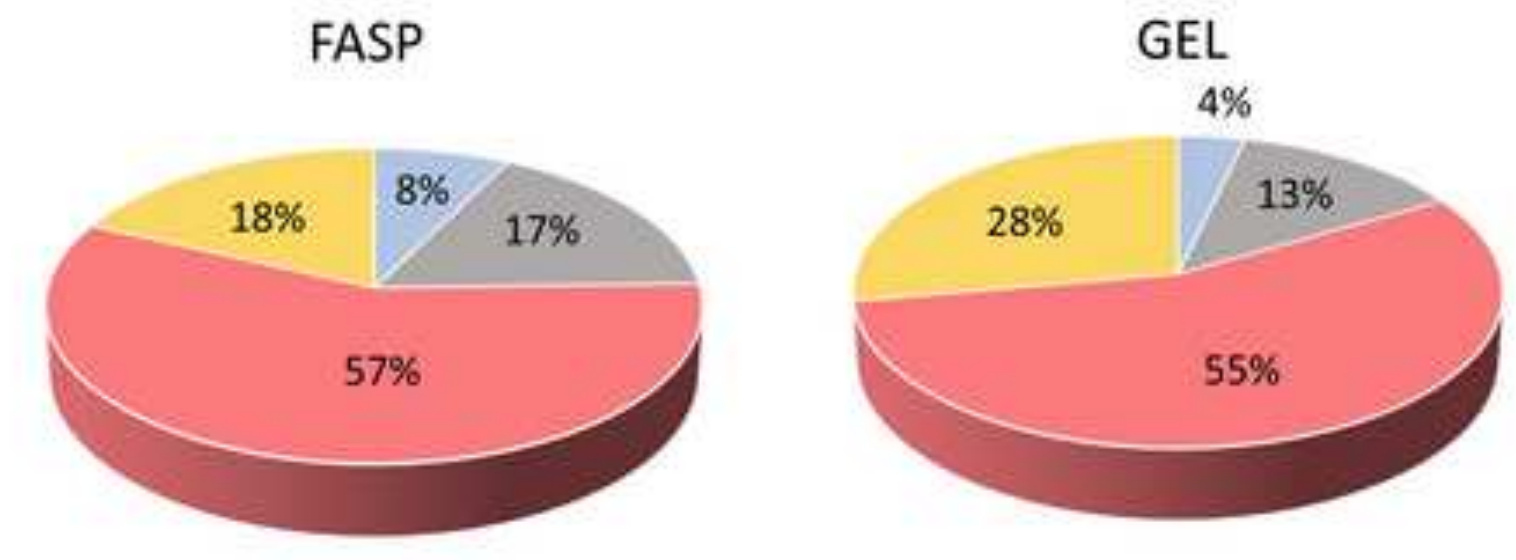

Signalling and cytoskeleton temodelling

Transmembrane transport

Structure maintenance

Other

B)

\section{SUBCELLULAR LOCATION}

FASP

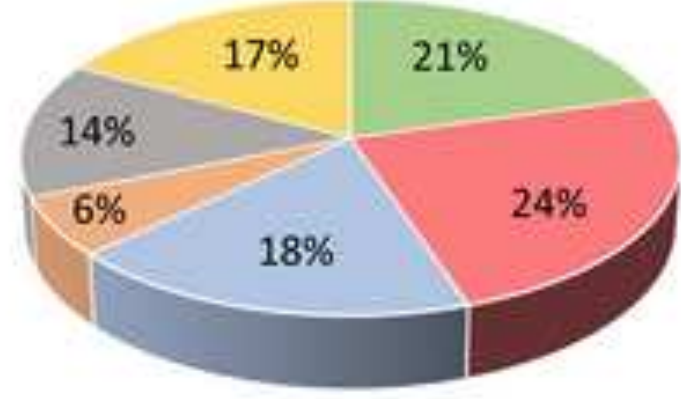

Membrane and cell surface 
Figure 4

Click here to download high resolution image

-

.

.
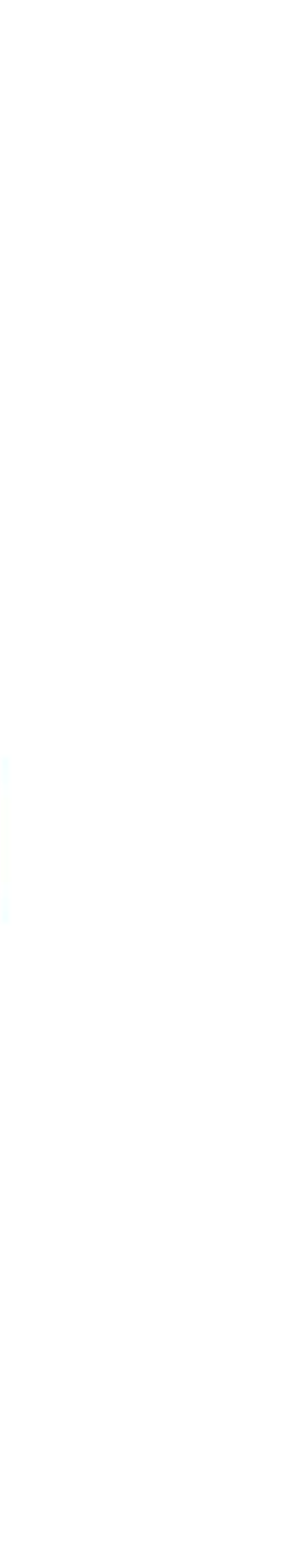

BASAL (51) GPVI (52)

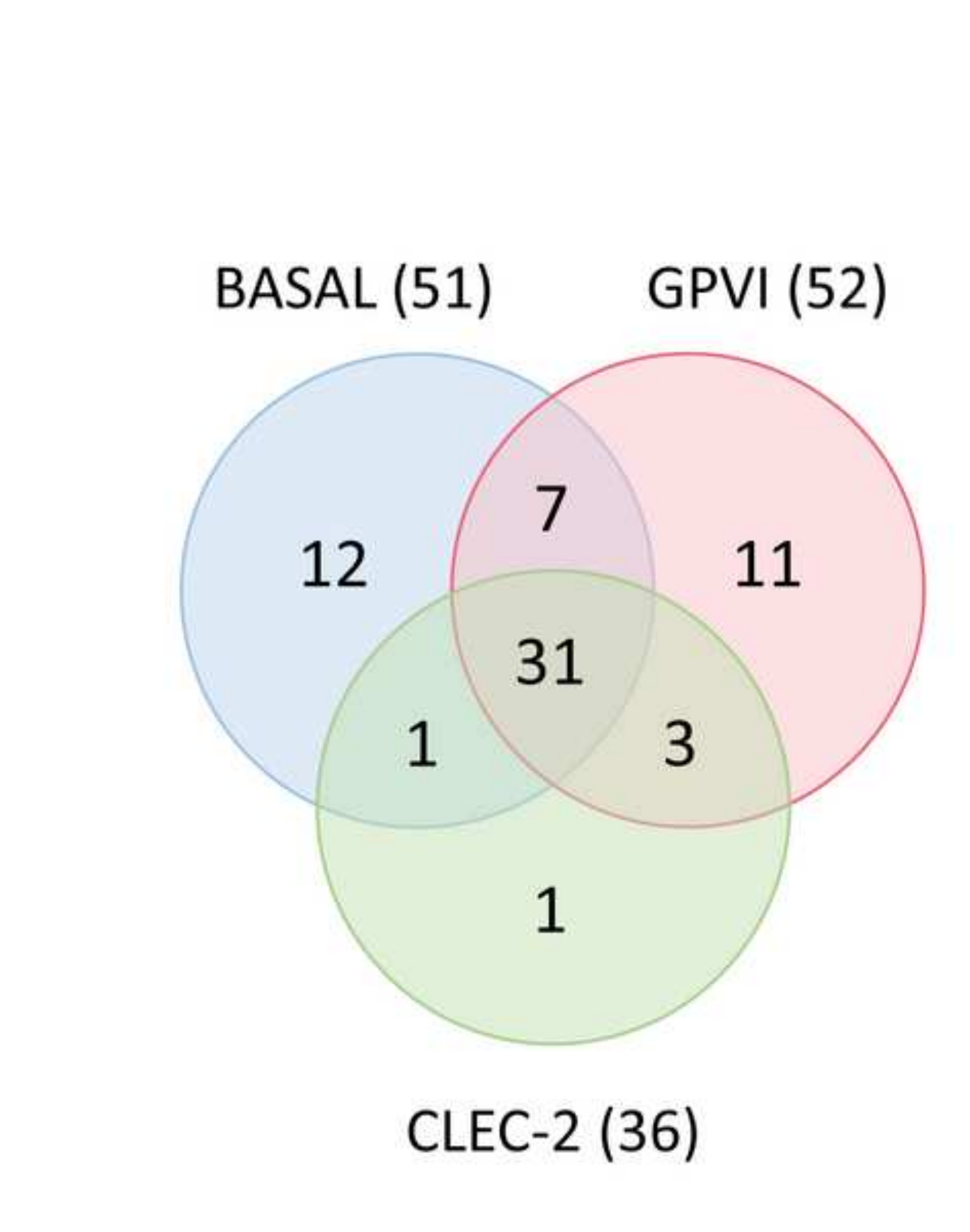

CLEC-2 (36)

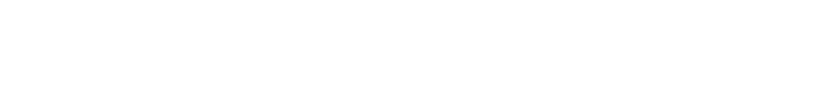

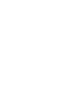

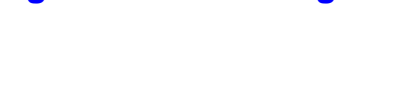
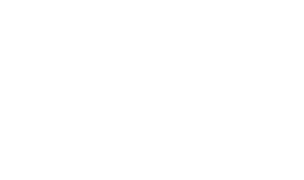
A)

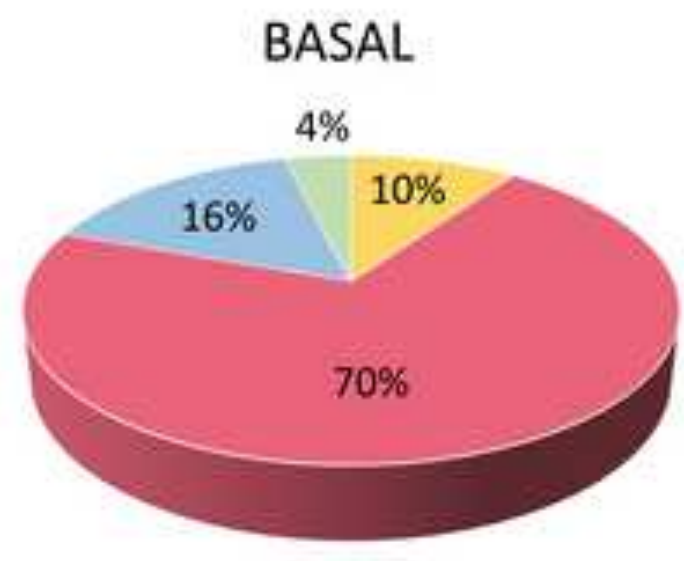

\section{FUNCTION}

GPVI

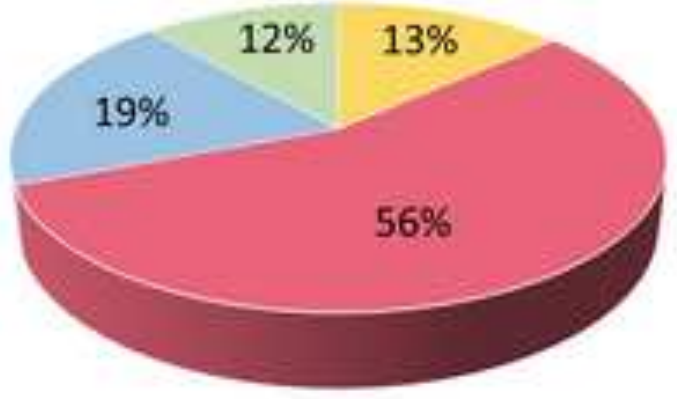

\section{CLEC-2}

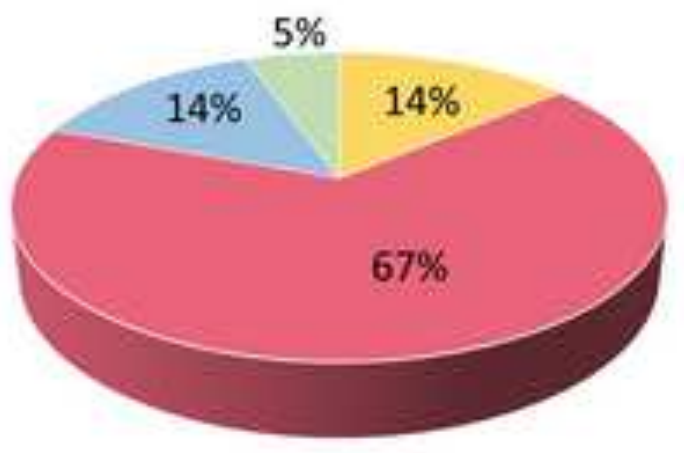

Signaling and cytoskeleton remodelling | Transmembrane transport 1 Structure maintenance Other

B)

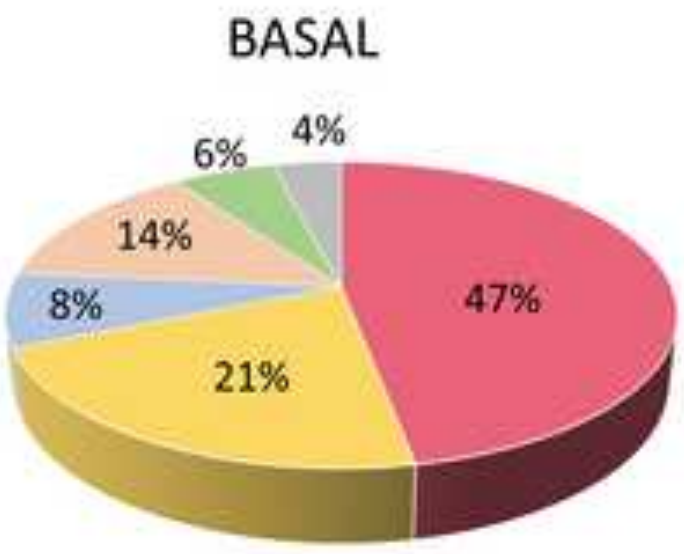

SUBCELLULAR LOCATION

GPVI

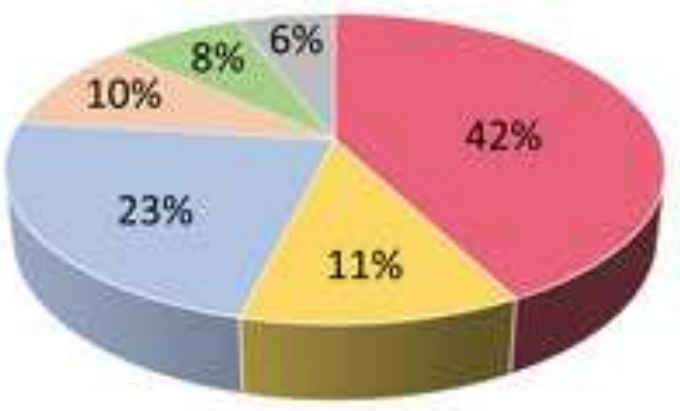

\section{CLEC-2}

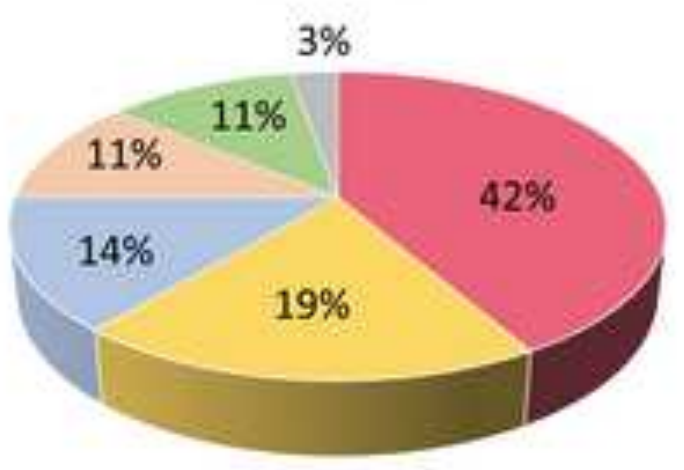


Click here to download high resolution image

A)

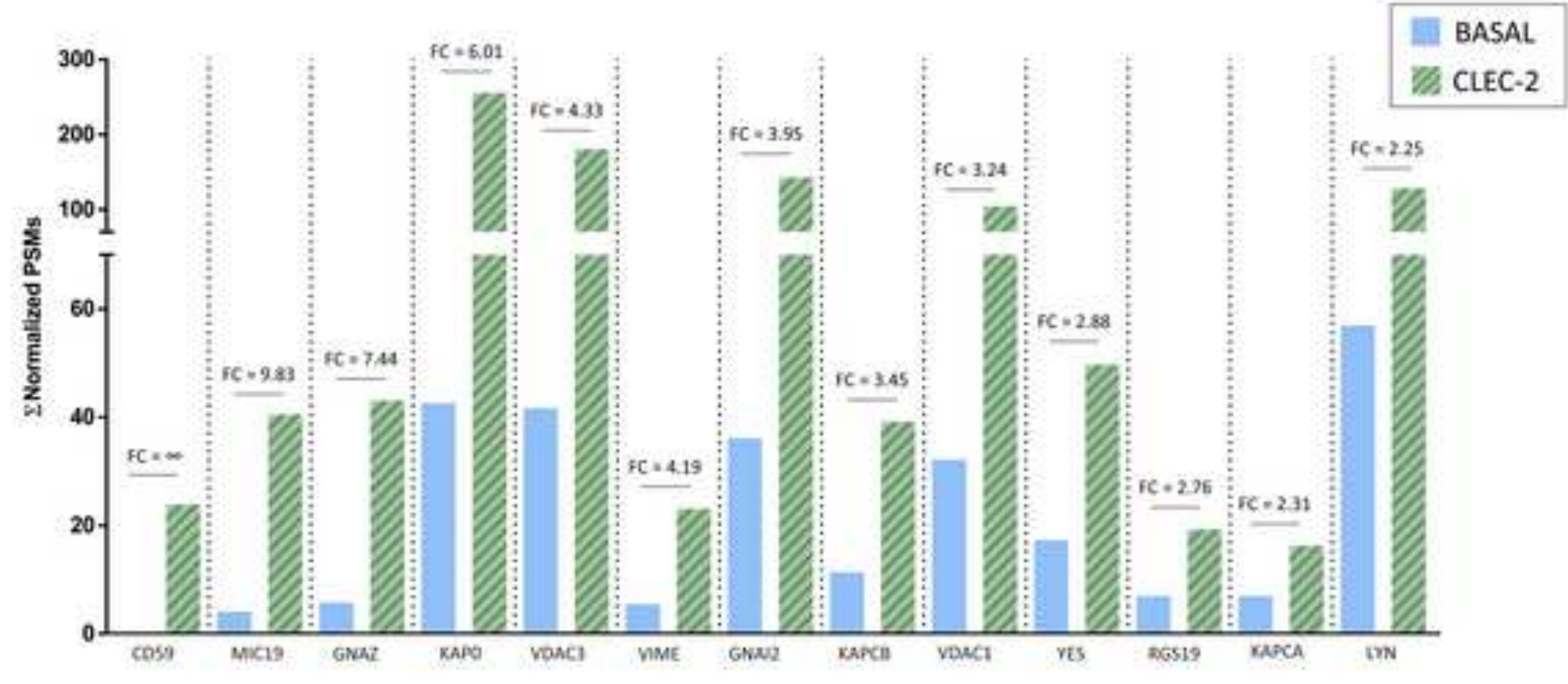

B)

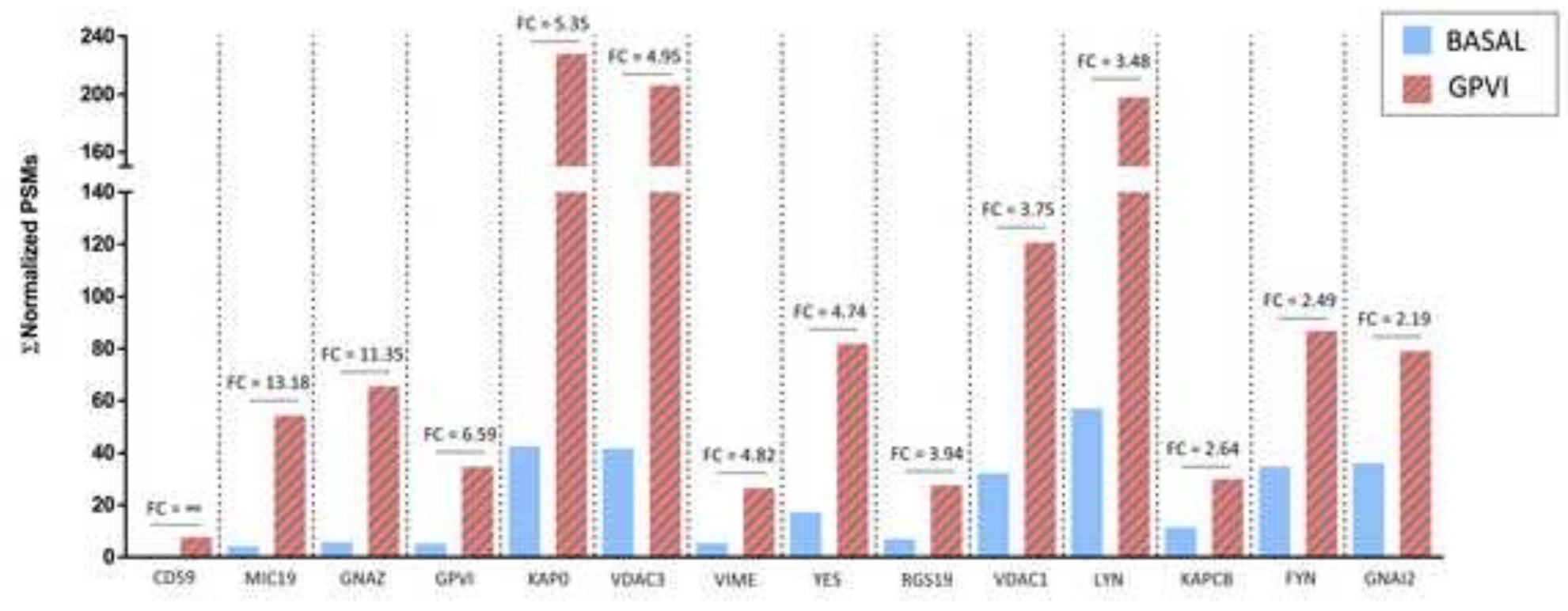


A)

$\begin{array}{lllllllll}1 & 2 & 3 & 4 & 5 & 6 & 7 & 8 & 9\end{array}$

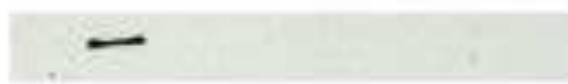

$\begin{array}{lllllllll}1 & 2 & 3 & 4 & 5 & 6 & 7 & 8 & 9\end{array}$

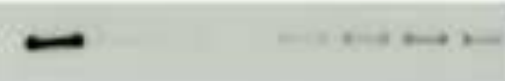

$\begin{array}{lllllllll}12 & 3 & 4 & 5 & 6 & 7 & 8 & 9\end{array}$ $\longrightarrow$ $\begin{array}{lllllllll}1 & 2 & 3 & 4 & 5 & 6 & 7 & 8 & 9\end{array}$

IB: Flot-1

$\begin{array}{lllllllll}1 & 2 & 3 & 4 & 5 & 6 & 7 & 8 & 9\end{array}$

- $\quad \ldots . . .18$ I8: Lyn

$\begin{array}{lllllllll}1 & 2 & 3 & 4 & 5 & 6 & 7 & 8 & 9\end{array}$

IB: Actin

B)

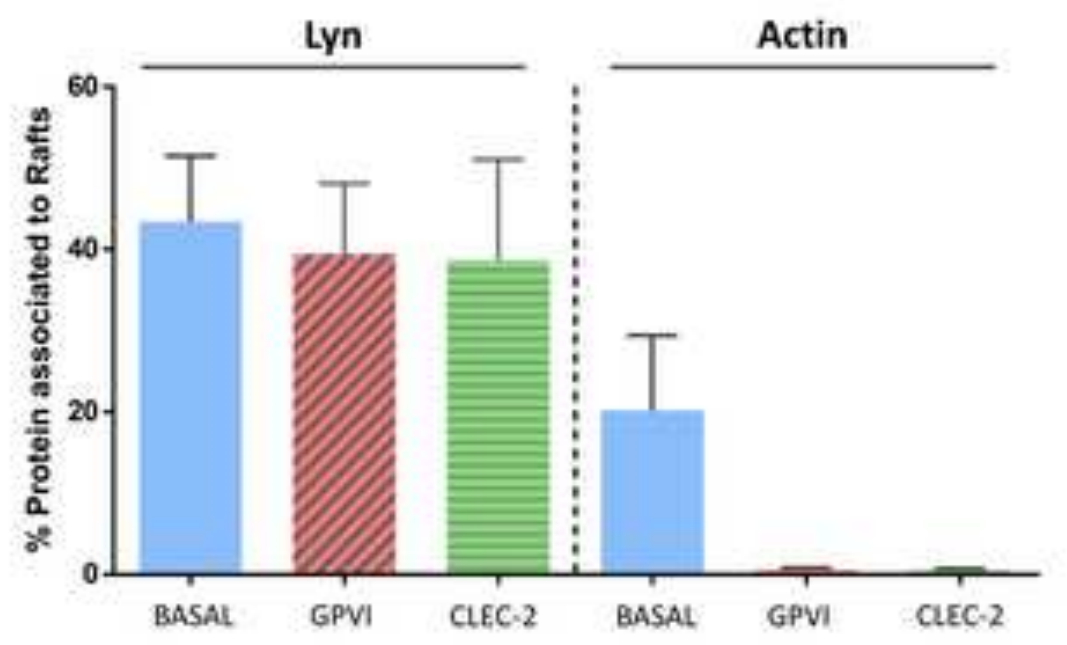




\section{Biological process}

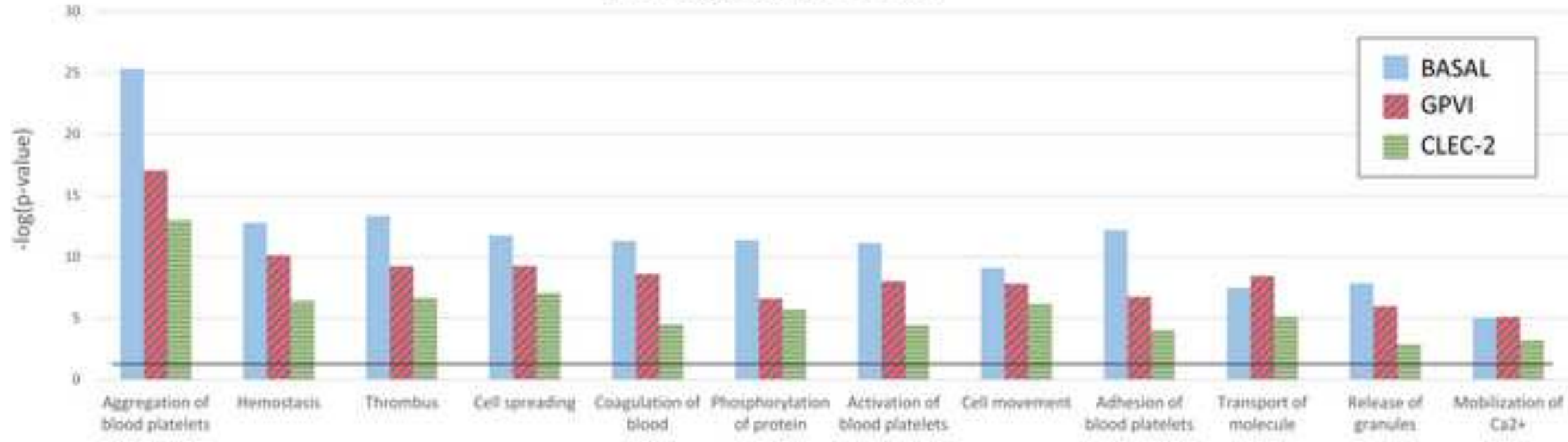

\section{Canonical pathway}

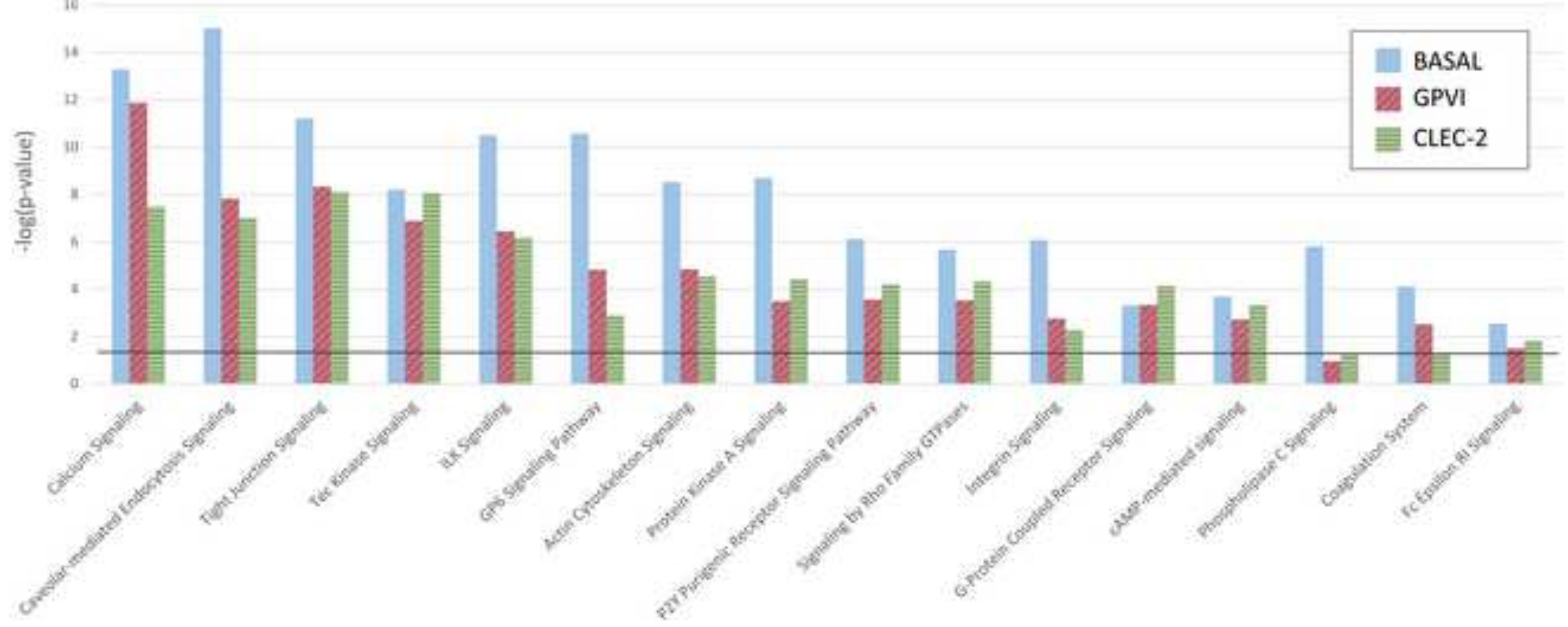


Supplementary table 1
Click here to download Supplementary material: Supplementary table 1.pdf

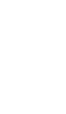

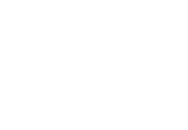
.

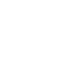
. (1)

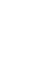
(1) (a)

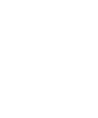

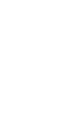

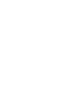

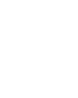

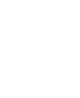
(1)

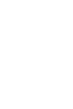
(1)

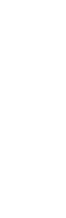
.

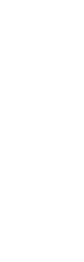
.

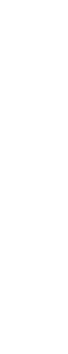

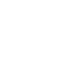

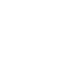
更 更 更 更 更 更 更 . 
Supplementary table 2
Click here to download Supplementary material: Supplementary table 2.pdf

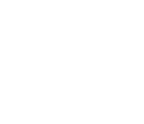
(1)

(1)
$\sqrt{2}$ or

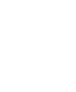

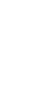
(1)

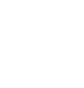
(1) (1) (1)

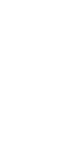

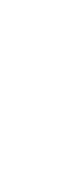
.

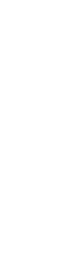

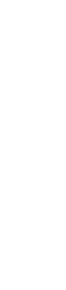
n

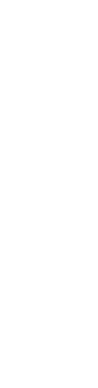

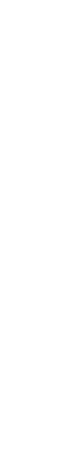

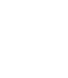
. 更 . 更 . 更 . 
Conflict of Interest

Click here to download Conflict of Interest: Conflict of interest.docx 